\title{
Parentalidad Postdivorcio: Características Relacionadas con la Asignación de la Custodia de Hijos e Hijas tras la Ruptura de Matrimonios Heterosexuales. El Caso de Andalucía
}

\section{Post-Divorce Parenting: Characteristics Related to the Assignment of Custody of Children after the Breakdown of Heterosexual Marriages. The Case of Andalusia}

\author{
José Manuel Jiménez-Cabello \\ josejicabello@ugr.es @0000-0001-7518-2134 \\ Inmaculada Puertas-Cañaveral \\ ipuertas@ugr.es@0000-0002-6299-7577 \\ José Luis Paniza-Prados \\ jpaniza@ugr.es@0000-0001-5397-8289
}

Departamento de Sociología, Universidad de Granada.

C/ Rector López Argüeta, s/n. 18001 Granada.

José Manuel García-Moreno

jmgarciamoreno@uma.es @ 0000-0003-2429-8764

Área de Sociología, Universidad de Málaga.

Campus El Ejido, s/n. 29071 Málaga.

\section{INFO ARTÍCULO}

Recibido: $17-06-2020$

Revisado: 12-11-2020

Aceptado: 05-12-2020

\section{PALABRAS CLAVE}

Divorcio

Custodia

Andalucía

Familia

Cuantitativo

Heterosexuales

\section{KEYWORDS}

Divorce

Custody

Andalusia

Family

Quantitative

Heterosexual

\section{RESUMEN}

La presente investigación se centra en identificar la relación de determinadas variables sociales con la asignación de la custodia tras el divorcio de matrimonios heterosexuales en Andalucía. Para ello se emplea metodología cuantitativa, utilizando la Estadística de Nulidades, Separaciones y Divorcios (INE, 2017) y datos de indicadores económicos como el Producto Interior Bruto (2017). Su explotación permite tener en cuenta todo un conjunto de variables necesarias para comprender el fenómeno objeto de estudio. Se realizan análisis de tipo descriptivo y multivariante (análisis factorial y de correspondencias). Los principales resultados obtenidos confirman que variables como el número de hijos menores o mayores dependientes, el fallo de la sentencia y el PIB de cada provincia, están relacionadas significativamente con la modalidad de custodia finalmente asignada. Otras variables como la edad de los cónyuges o su nacionalidad no parecen tener asociación en la modalidad que se otorga. 


\begin{abstract}
obtained confirm that variables such as the number of dependent minor children or dependent children of legal age, the judgment failure and the GDP of each province are significantly related to the custody modality finally assigned. Other variables such as the age of the spouses or their nationality do not seem to have an association in the modality that is granted.
\end{abstract}

\title{
1. INTRODUCCIÓN
}

La sociedad española es el reflejo de cómo los procesos de ruptura matrimonial se han ido aceptando poco a poco, como elemento caracterizador de trayectorias vitales que se modifican y se adaptan a nuevas situaciones. Esa aceptación progresiva de la ruptura matrimonial como opción de vida, ha ido pasando por diversas etapas que comprenden desde una visión estigmatizadora hasta entenderla como una solución a un proyecto de vida en común insatisfactorio. En pocos años se ha ido consolidando una aceptación amplia y sin obstáculos que incluso tuvo una plasmación legal en el cambio de la Ley del divorcio (Becerril, 2008). Es necesario resaltar como la Ley 15/2005, de 8 de julio por la que se modifican el Código Civil y la Ley de Enjuiciamiento Civil en materia de separación y divorcio, supuso un gran avance en esta materia. Sobretodo trajo consigo pasar de un modelo de ruptura que obligaba a pasar por diversas etapas, a otro en el que se accede al divorcio sin dicha condición y donde además los plazos temporales se acortan.

En términos absolutos, los divorcios en España se sitúan en torno a los 100.000 procesos cada año, si bien desde 2013 están por debajo de esa cifra, alcanzando en 2018 un total de 95.254 procesos (INE, 2019). En Andalucía, concretamente, hubo 17.772 divorcios en 2018, situándose la tasa bruta de disoluciones en 2,11 por mil. Así, en esta Comunidad Autónoma (C.A., en adelante) se produce, prácticamente, un quinto del total de los divorcios acaecidos en España. Estos datos dan una idea de la dimensión y del reto que en Andalucía implican los procesos de divorcio a los que, sorprendentemente, poco análisis se ha dedicado.

El divorcio conduce a los cónyuges a un proceso de negociación que puede adoptar un carácter consensuado o contencioso. También, puede comenzar siendo contencioso y transformarse en consensuado. De entre todas las variables que se puedan relacionar con el carácter del proceso de divorcio, destaca una sumamente importante: la tenencia o no de hijos. Esta variable se erige en elemento decisivo que condiciona la ruptura. En el momento en que los matrimonios divorciados cuentan con hijos la situación se vuelve más compleja y la solución a la custodia de los menores constituye un proceso con múltiples aristas (Suso et al., 2012). La decisión en torno a quien corresponde la custodia de los hijos, y todos aquellos aspectos colaterales derivados de ella, conforman uno de los aspectos más polémicos que todo proceso de ruptura conlleva (Becerril, 2014). Al hacer referencia a la custodia y guardia de los hijos no se contempla únicamente el hecho compartir más o menos tiempo, sino que condiciona fundamentalmente, el uso de la vivienda familiar y la asignación de pensiones alimenticias (Becerril, 1999).

La custodia de los hijos e hijas hace referencia al cuidado diario, y remite por tanto a la coresidencia y al tiempo y al espacio compartido entre cada progenitor y sus hijos menores (Solsona et al., 2017). También puede entenderse como una situación jurídica que se da cuando un tribunal otorga, mediante sentencia, la guardia y custodia de los hijos menores de edad a uno o a ambos progenitores (Prieto, 2014). Es precisamente, de nuevo, la Ley 15/2005 la que introdujo un aspecto novedoso en cuanto a la asignación de la custodia: establece, por primera vez, en el derecho de familia español la posibilidad de ejercer la custodia compartida tras la ruptura (Catalán et. al., 2007). De tal forma, en España existen diversas modalidades de custodia: custodia exclusiva, compartida y a otros familiares (Catalán, 2011).

En España los procesos de divorcio que incluyen hijos o hijas comprenden más de la mitad de las rupturas. Datos de la Estadística de Nulidades, Separaciones y Divorcios (ENSD en adelante), indican que en 2018 los divorcios con hijos suponían un 57,4\%, siendo destacable especificar que desde 2007 este dato siempre supera el 50\%. En Andalucía este porcentaje es bastante parecido situándose, en los últimos años, en torno al 53-56\% representando una parte importante de los divorcios. En la esfera europea los datos son parecidos a los de España y Andalucía. En Portugal más de la mitad de las rupturas matrimoniales se producen con 
la presencia de hijos (Statistics Portugal, 2018) mientras que, en Francia, también país de nuestro entorno cercano, 6 de cada 10 divorcios involucran a varios menores, según datos del Ministére de la Justicie (2018).

Dada la posición que ocupa Andalucía respecto al total de divorcios en España, y tomando en consideración que en más de la mitad de los procesos en esta comunidad hay asignación de custodia de hijos, resulta imprescindible abordar esta realidad social con el fin de obtener conocimiento sobre un ámbito que no ha recibido atención empírica.

Es por esto que el presente artículo plantea como objetivo principal de investigación identificar si determinadas variables sociales tienen relación con la modalidad de custodia otorgada. Para ello, en primer lugar, se describe una panorámica de la evolución del divorcio y la custodia de los hijos en Andalucía; en segundo término, se realiza una revisión de la literatura en torno a las variables sociales que influyen en la asignación de la custodia; tercero, se implementa un análisis factorial y de correspondencias con las variables sociales seleccionadas para el caso andaluz; y por último se sintetizan las principales conclusiones alcanzadas.

\section{EVOLUCIÓN Y DATOS DEL DIVORCIO Y DE LA CUSTODIA DE LOS HIJOSEN ANDALUCÍA: LAIMPORTANCIA DE LA LEY DE 2005}

Después casi cuatro décadas desde la ley del divorcio de 1981, existe un trayecto extenso y sólido que permite estudiar la transformación que han ido sufriendo las rupturas matrimoniales en Andalucía. Este periodo ha sido bastante intenso en lo referido a los cambios familiares $y$, en general, a las transformaciones que ha sufrido la sociedad española (Becerril, 2015; Meil, 2015). Son numerosos los aspectos que han tenido influencia en este periodo, entre los que se pueden destacar los culturales, económicos, demográficos y políticos (Ayuso, 2019).

La legislación del divorcio en España, y por tanto en Andalucía, ha experimentado determinadas modificaciones legales, lo que nos permite analizar el impacto que las mismas han tenido. A finales de los años setenta del siglo pasado se inicia cierta tradición, de estudios referidos al divorcio, con posibilidades limitadas de datos e incluso referidos a la Segunda República (Alberdi, 1979; Lezcano, 1979). Esto tendrá escasa continuidad en los ochenta (Borrajo, 1989; López \& Toharia, 1989). En la década de los noventa existen estudios, bastante completos, sobre la biografía del divorcio, así como de las consecuencias que supone (Becerril, 1999). Habrá que esperar algunos años para los primeros análisis sobre el divorcio que se basen en una fuente más moderna, la Estadística de Nulidades, Separaciones y Divorcios (ENSD, en adelante) del Instituto Nacional de Estadística.

Aun así, será la entrada en vigor de la Ley 15/2005 de 8 de julio la que genera el cambio más actual. Esta reforma de la Ley inicial del divorcio de 1981, es la más significativa que ha tenido lugar. A pesar de ello, el impacto de esta reforma no ha tenido el seguimiento y atención necesario, aunque comienzan a aparecer estudios centrados en ella. Así, se cuenta con un análisis muy completo sobre la evolución del divorcio realizado por Solsona y Simó (2007), donde se estudia desde una amplia perspectiva las fuentes, datos y tendencias que el divorcio presentaba hasta esa fecha. En cualquier caso, las investigaciones existentes han puesto el foco, principalmente, en las causas de la ruptura matrimonial y la evolución de la misma (Becerril, 2015; Solsona \& Simó, 2007) o se han centrado en aspectos jurídicos de funcionamiento (Ruiz \& Alcázar, 2017). Más actual, es la investigación de Becerril y Jiménez (2019), donde se determina y concreta el impacto de la ley de 2005.

La relevancia de la Ley 15/2005 reside, fundamentalmente, en la introducción de la posibilidad de divorciarse sin necesidad de separación previa, ni de tener que demostrar alguna causa justificable. Esto no excluye los procesos de separación, que sin embargo no permanecen como un trámite obligatorio y previo al divorcio, sino como una opción junto a este.

Otro cambio a tener en cuenta es la reducción de los plazos mínimos requeridos a la duración del matrimonio, que de un año pasa a tres meses, o incluso menos si hay riesgo en la integridad, libertad o indemnidad sexual del demandante, hijos u otros miembros familiares (Becerril \& Jiménez, 2019). La importancia, y determinación, de esta ley es bastante destacable (ver figura 1). El año 2005 supone un momento de "ruptura": los 
divorcios aumentan de forma considerable, tanto en España como en Andalucía, pero no como resultado de un aumento real de las rupturas en general, sino debido a la modificación del ordenamiento jurídico. El divorcio crece en detrimento de las separaciones que desde entonces han ido descendiendo hasta prácticamente presentar valores residuales hoy día.

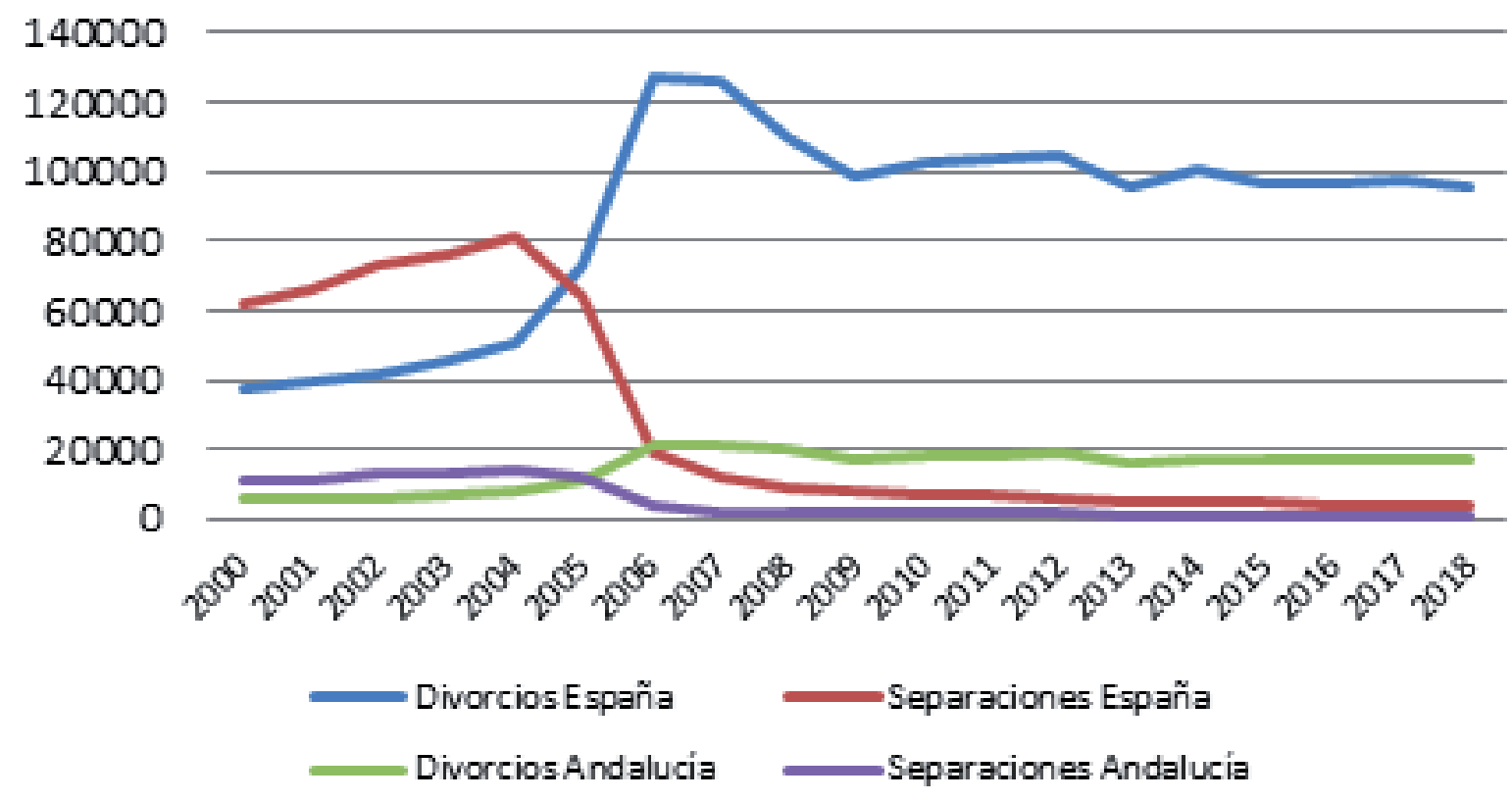

Figura 1. Evolución de la ruptura matrimonial en España y Andalucía (en miles). Fuente: Elaboración propia a partir de la ENSD.

En términos absolutos, los divorcios en España se sitúan en torno a los 100.000 procesos cada año, si bien desde 2013 están por debajo de esa cifra, alcanzando en 2018 un total de 95.254 procesos (INE, 2019). Tales cifras determinan que el indicador de divorcio de España sea de 2,13 divorcios por cada mil habitantes. La misma está algo por encima de la media de Europa $(1,9)$ y es menor que las registradas por países como Lituania $(3,1)$, Dinamarca $(3,0)$ o Finlandia y Suecia $(2,5$ y 2,4 respectivamente). Además, es un indicador que supera a muchos otros países, incluidos algunos nórdicos (Noruega, con 1,9), y a todos los de nuestro entorno más inmediato en términos socioculturales, como Grecia $(1,0)$, Francia $(1,9)$ o Italia $(1,6)$ (EUROSTAT, 2018). Por tanto, no es baladí la cuestión del divorcio en nuestro país. La información de este indicador (que puede verse afectado por las oscilaciones propias de los matrimonios) puede ser completada comparando los matrimonios con los divorcios. Así, cada año por cada 100 matrimonios se producen 58 divorcios en España. Si tuviéramos en cuenta esta medida, seríamos el segundo país de la Europa de los 27 con mayor divorcio, solo superados por Luxemburgo, con 100 matrimonios por cada 65 divorcios. Con estos datos, parece claro que el estudio del divorcio en España, es un campo de estudio que merece la atención de la comunidad científica.

Por su parte, en Andalucía, hubo 17.772 divorcios en 2018, situándose la tasa bruta de disoluciones en 2,11 por mil, valor muy parecido a la media nacional. Así esta comunidad ocupa, prácticamente, un quinto del total de los divorcios acaecidos en España. Estos datos dan una idea de la dimensión y del reto que en Andalucía implican los procesos de divorcio. Esto puede visualizarse de forma más nítida observando lo que ha sucedido por provincias desde una fecha anterior (figura 2), teniendo en cuenta las diferencias que se producen atendiendo al tamaño de la población de cada una de ellas. De nuevo se advierte que el momento 
de cambio se produce en el periodo 2005-2006. Como se indicó con anterioridad, esto no implica que las rupturas aumentasen, sino que el incremento del divorcio se produce como modalidad, en detrimento de la separación, y no como resultado del aumento de rupturas.

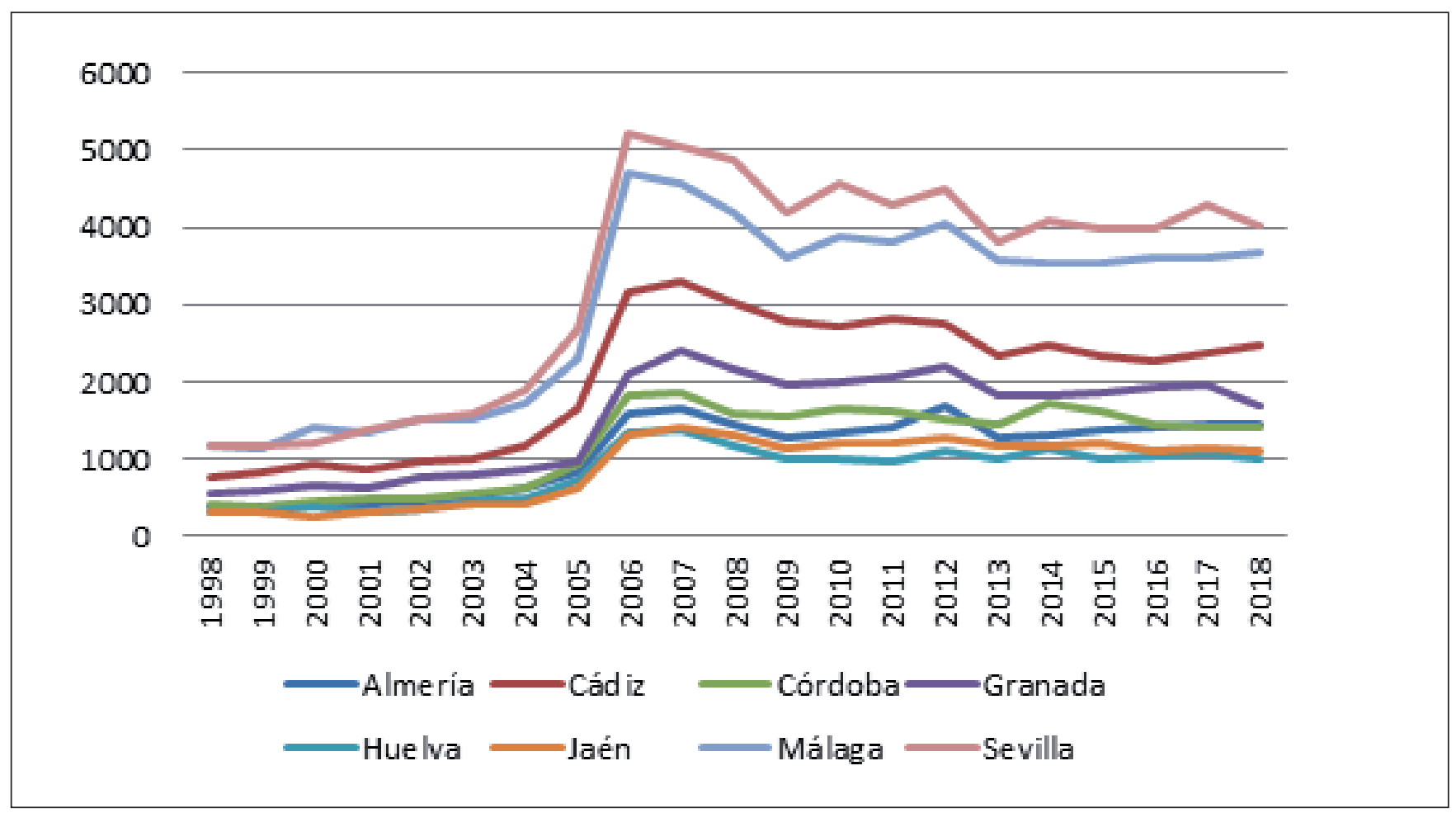

Figura 2. Evolución del divorcio en Andalucía (en miles). Fuente: Elaboración propia a partir de la ENSD.

Más allá de las cifras de divorcios y de la posición relativa en que se encuentra España, y Andalucía en particular, resulta interesante conocer cómo se producen estos divorcios, ya que el carácter consensuado o contencioso de los mismos será determinante para analizar el aspecto que aquí interesa en mayor medida: la asignación de la custodia de los hijos (Wilcox et al., 1998; Juby et al. 2005).

Si se analiza el fallo de las sentencias (tabla 1 ) se puede concluir como el porcentaje de resoluciones consensuadas ha aumentado en Andalucía, ligeramente, desde 2012 hasta 2018 (un 5,8\%). Aun así, es de destacar que el porcentaje de procesos consensuados de esta comunidad se encuentra por debajo de la media nacional (76\% según la ENSD). Este suave aumento en la cantidad de divorcios consensuados ha sido una constante común a nivel nacional representando, aproximadamente, dos tercios del total (Flaquer, 2012). Los datos más recientes de la ENSD a nivel nacional y autonómico confirman, y reafirman al alza, lo concluido por Flaquer (2015) ya que en 2018 los divorcios consensuados suponen un $76 \%$ del total, mientras que por ejemplo en 2010 era un 67,4\%.

En cuanto a la asignación de la custodia de los hijos, con la ya mencionada ley de 2005, la aparición de la figura de la custodia compartida normalizaba una situación que en nuestro entorno europeo ya había sido reconocida (Alemania en 1997, Holanda en 1998, Austria en 2001 o Francia en 2002), si bien en España esta figura se introdujo de una forma tímida e incluso inconstitucional en lo referente a la necesidad del informe positivo del fiscal. Pero, en cualquier caso, desde entonces se incrementó el debate sobre la idoneidad de la modalidad compartida y, por tanto, sobre la situación en la que quedan los hijos menores tras la ruptura matrimonial (Becerril, 2014; Flaquer, 2012; Torres-Perea, 2011).

Esta nueva situación, pocos años después de la Ley de 2005, permitió que distintas CC.AA. (Aragón, Cataluña, Comunidad Valenciana, Navarra y País Vasco) comenzasen a legislar sobre las situaciones y las relaciones con los menores tras el divorcio (Solsona et al., 2017). En la segunda década de este siglo, se inicia entre 
Tabla 1. Tipo de proceso de divorcio según fallo de la sentencia en Andalucía según provincia (2012-2017).

\begin{tabular}{|c|c|c|c|c|c|c|c|c|c|c|c|c|c|c|}
\hline \multirow{2}{*}{ Provincia } & \multicolumn{2}{|c|}{2012} & \multicolumn{2}{|c|}{2013} & \multicolumn{2}{|c|}{2014} & \multicolumn{2}{|c|}{2015} & \multicolumn{2}{|c|}{2016} & \multicolumn{2}{|c|}{2017} & \multicolumn{2}{|c|}{2018} \\
\hline & Cons & No & Cons & No & Cons & No & Cons & No & Cons & No & Cons & No & Cons & No \\
\hline Almería & 72,1 & 26,9 & 73,2 & 26,7 & 77,8 & 22,2 & 67,2 & 23,8 & 71,6 & 28,4 & 74,3 & 25,7 & 74,5 & 25,5 \\
\hline Cádiz & 64,7 & 34,3 & 65,5 & 34,5 & 65,6 & 34,4 & 67 & 33 & 69,4 & 30,6 & 74,2 & 25,8 & 74,3 & 25,7 \\
\hline Córdoba & 63,9 & 36,1 & 64,4 & 35,6 & 58,7 & 41,3 & 62,8 & 37,2 & 70,0 & 30,0 & 66,9 & 33,1 & 70,0 & 30,0 \\
\hline Granada & 64,1 & 35,9 & 64,4 & 35,5 & 71.9 & 28,1 & 69,1 & 30,9 & 69,4 & 30,6 & 72,9 & 27,1 & 73,2 & 26,8 \\
\hline Huelva & 58,0 & 42,0 & 57,7 & 42,3 & 56,8 & 43,2 & 64,6 & 35,4 & 64,0 & 36,0 & 64,5 & 35,5 & 63,8 & 36,2 \\
\hline Jaén & 65,6 & 34,4 & 65,5 & 34,5 & 71,6 & 28,2 & 73,7 & 26,3 & 75,4 & 24,5 & 75,5 & 24,5 & 75,7 & 24,3 \\
\hline Málaga & 72,1 & 27,9 & 73,5 & 26,5 & 74,8 & 25,2 & 73,4 & 26,6 & 75,8 & 24,2 & 78,2 & 21,8 & 78,9 & 21,1 \\
\hline Sevilla & 73,9 & 26,1 & 73,8 & 26,2 & 75,0 & 25,0 & 72,0 & 28,0 & 70,6 & 29,4 & 69,5 & 30,5 & 70,1 & 29,9 \\
\hline And. & 66,8 & 32,9 & 67,3 & 32,73 & 69,1 & 30,9 & 68,8 & 30,2 & 70,8 & 29,2 & 72,0 & 28,0 & 72,6 & 27,4 \\
\hline
\end{tabular}

Fuente: Elaboración propia a partir de la ENSD.

las CC.AA. ${ }^{1}$ cierta carrera por tener una ley autonómica propia que, sin duda, ha impulsado el debate y ha mantenido vivo el reto de regular y conocer las dinámicas familiares y los tipos de custodia. La reforma de 2005, junto a las de legislaciones autonómicas que se han ido impulsando, hace posible que por primera vez en España el modelo de monoparentalidad en la custodia sea cuestionable y pueda surgir una alternativa, no tanto en la monoparentalidad masculina (lo que supondría regresar a la situación de partida) como en el reconocimiento de la custodia compartida como opción.

En Andalucía (ver figura 3) la custodia compartida se encuentra muy por debajo de la media nacional, siendo la diferencia de un 10,5\% en 2018. Además, en este caso, se está analizando una comunidad carente de ordenamiento jurídico propio en materia de asignación de custodia, por lo que cabría esperar que el porcentaje de custodias compartidas sea similar al de aquellas CC.AA. que tampoco la poseen. Sin embargo, puede verse como Andalucía también se encuentra, con respecto al porcentaje de custodia compartida, por debajo del conjunto de CC.AA. sin legislación propia, con una diferencia de un 5,4\%.

Precisamente, en relación a la puesta en marcha de la autoregulación de la custodia compartida, existe cierto debate en esta C.A. El Pleno del Parlamento andaluz aprobó, el pasado 4 de abril de 2020, un punto de una proposición no de ley impulsada por la formación política Ciudadanos mediante la que se reclama que el Consejo de Gobierno inste al Ejecutivo central a reformar la Ley de Custodia Compartida a fin de que esta modalidad, en caso de ruptura, sea la más deseable y normal. En virtud de la iniciativa, la cámara de representantes andaluza solicitó que se impulsará la reforma de la referida norma para que quedara recogida y, en consecuencia, se siguiera la doctrina actual del Tribunal Supremo (TS) que determina este tipo de custodia como la más deseable siendo, además, la que permite en mayor medida el respeto al interés superior del menor ${ }^{2}$.

1. Las CC.AA. que poseen legislación propia en este momento son: Aragón, Cataluña, País Vasco y Navarra. En la Comunidad Valenciana, tras un proceso de suspensión y nueva puesta en marcha, fue derogada definitivamente por el TC.

2. El interés superior del menor hace referencia a la realidad de niños y niñas como sujetos dignos de atención, promoción, provisión y protección. Este criterio ha de aplicarse en todas aquellas situaciones o conflictos donde se hallen involucrados menores de edad (Ravetllat, 2012). 


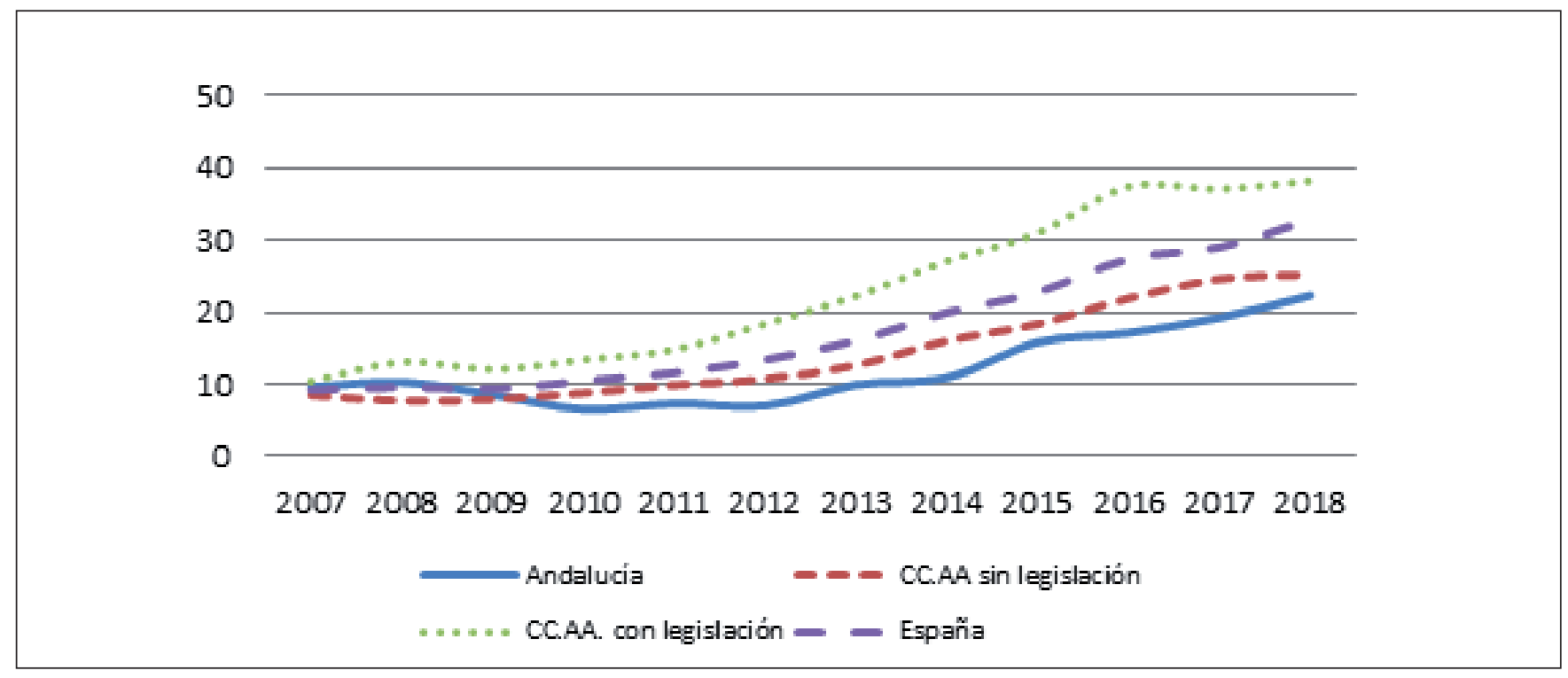

Figura 3. Evolución de las custodias compartidas en España, Andalucía y por conjuntos según la existencia de ordenamiento propio (\%). Fuente: Elaboración propia a partir de la ENSD.

\section{ANÁLISIS DE LAS VARIABLES SOCIALES INFLUYENTES EN LA ASIGNACIÓN DE LA CUSTODIA}

El análisis sobre la asignación de la custodia, y el desarrollo de estas, es completo a nivel nacional e internacional desde diversos ámbitos como la sociología (Ruíz y Alcázar, 2017; Solsona et al., 2017 entre otros trabajos) o el derecho (Morillas, 2017; Prieto, 2014; Torres-Perea, 2011 o Viñas, 2011). Sin embargo, a nivel andaluz no se disponen de investigaciones pormenorizadas relativas a esta temática, de ahí la necesidad de aproximarnos a este objeto de estudio. Para ello, tras la oportuna revisión de la literatura, se han identificado diversas variables que están en relación con el tipo de custodia finalmente asignada y que pueden ayudar a conocer por qué se da una modalidad u otra de custodia en Andalucía.

En este sentido, el número de hijos ha sido bastante analizado, al igual que estos sean menores o mayores dependientes. La custodia compartida, parece mostrarse con más probabilidades en aquellas situaciones con dos o más hijos siendo menos frecuente con uno solo (Jiménez \& Becerril, 2020; Becerril, 2015; Spijker, 2012). Por su parte, Cancian y Meyer (1998) indican que en el posible caso de que existan varios hijos tras del proceso de divorcio, los padres están de acuerdo en no separar a los hermanos en el $97 \%$ de los casos. Así, en sólo el 3\% de los juicios con varios hijos menores de edad y con el acuerdo de los padres, algunos de ellos terminarán viviendo con su madre, mientras que otros lo harán con su padre. Por el contrario, Maccoby y Mnookin (1992) indican que la compartida es más probable en parejas con un solo hijo. En cuanto a la edad concreta de los hijos, Cancian y Meyer (1998) no encontraron ninguna relación determinante entre esta y la modalidad de custodia asignada, pero Maccoby y Mnookin (1992) observaron que los niños con edades comprendidas entre 2 y 9 años de edad, tienen más probabilidades de tener un régimen de custodia compartida que exclusiva.

El fallo de la sentencia parece ser otro de los factores determinantes en la asignación de la custodia, ya que en gran medida refleja la más que posible relación entre cónyuges y como pueda ser esta en un futuro. Esto, ha sido comprobado por algunos autores en otros países, obteniendo que la custodia compartida será más probable cuando se experimenta baja conflictividad entre los miembros de la pareja (Wilcox et al., 1998; Chausseborg et al., 2009). Por ejemplo, el $81 \%$ de las custodias compartidas que se otorgan en Francia son por acuerdo (Jousselme, 2008).

Otro aspecto fundamental se asienta sobre la edad de los miembros de la pareja. La tendencia general observada es que la probabilidad de que exista una custodia compartida es bastante alta en madres muy jóvenes, descendiendo en las madres veinteañeras y en los primeros años de la treintena, para posteriormente, ascender a partir de los 33 años en adelante (Cancian \& Meyer, 1998; Juby et al., 2005; Solsona et al., 2014). 
En cuanto a la nacionalidad de los cónyuges, Becerril y Jiménez (2016), comprobaron como la nacionalidad de las personas implicadas en la disolución matrimonial es una variable a tener en cuenta.

Igualmente, parece influir en la modalidad de custodia que se asigna, el sexo de la persona que interpone la demanda de divorcio. Así, por ejemplo, Brinig y Allen (2002) concluyen que si la mujer solicita el divorcio suele tener, en mayor medida, la custodia monoparental. Igualmente señalan que el mecanismo es, más bien: que la mujer demanda el divorcio, mayoritariamente, porque tiene más probabilidades de quedarse con la guarda y custodia. Estos mismos autores examinaron distintos factores, como el poder en la pareja o las ganancias económicas. Concluyen que, de todos los factores, la posibilidad de quedar con los hijos es uno de los más determinantes para solicitar el divorcio. En este sentido se apunta a cómo las relaciones de género son, igualmente, fundamentales. Una mayor igualdad o simetría en las relaciones de poder está asociada con mayor probabilidad de establecer la modalidad compartida mientras que si esta es desigual o asimétrica predomina la exclusiva (Solsona et al., 2016).

Por último, resultan significativas las variables relacionadas con los aspectos de carácter económico. De hecho, la custodia compartida es más probable cuando ambos padres realizan un trabajo remunerado (Juby et al., 2005) siendo la exclusiva más propia cuando sólo uno de ellos trabaja (Cancian \& Meyer, 1998). Para el caso de estudio que nos atañe, Andalucía, hay que señalar que con los datos de los que se dispone no es posible medir si el nivel de ingresos de ambos cónyuges, en el caso de que los haya, sea una variable significativa en la asignación de la custodia como apuntan las investigaciones referidas anteriormente (Juby et al., 2005; Cancian \& Meyer, 1998). Igualmente sucede con las tasas de empleo, desempleo y actividad de varones y mujeres. Por tanto, procederemos a comprobar si el aspecto económico es determinante mediante otro tipo de indicador como el Producto Interior Bruto de cada provincia. Este, ya ha sido utilizado en algunas investigaciones relativas a la asignación de la custodia (Becerril, 2012). Advertir que, aunque este es un indicador muy general, está relacionado con una mayor o menor tasa de empleo, una variable clave para la custodia al estar ligada con la capacidad de sustentabilidad de los progenitores.

Tras esta revisión de la literatura, las variables ${ }^{3}$ consideradas para el presente estudio son las siguientes: la existencia de hijos menores o mayores dependientes y el número de estos, el fallo de la sentencia, la edad y nacionalidad de los miembros de la pareja, el cónyuge demandante del proceso y el producto interior bruto (PIB) de cada provincia (variable socioeconómica).

\section{METODOLOGÍA}

Tras la revisión de la literatura realizada, y puesto que nuestro objetivo es averiguar si determinadas variables sociales están relacionadas con la asignación de la custodia en Andalucía, se establece como hipótesis de investigación la siguiente: la tenencia y número de hijos menores y mayores dependientes, la edad de los miembros de la pareja y el fallo de la sentencia son variables relacionadas con la asignación de la modalidad de la custodia de los hijos tras el divorcio de matrimonios heterosexuales en Andalucía.

La metodología empleada para contrastar dicha hipótesis es de carácter cuantitativo, utilizando para ello datos secundarios procedentes de la base de datos Estadística de Nulidades, Separaciones y Divorcios (ENSD), que nos ha sido facilitada por el Instituto Nacional de Estadística (INE).

Esta es una estadística de carácter anual realizada por el INE en virtud de un acuerdo con el Consejo General del Poder Judicial. Su elaboración se realiza a partir de la información sobre sentencias que proporcionan distintos órganos judiciales competentes en esta materia. La base de datos considerada ha sido filtrada para Andalucía, obteniendo 17.437 casos de divorcio de matrimonios heterosexuales (sobre un total de 102.339 casos para toda España), para el año $2017^{4}$.

3. Se han analizado las variables seleccionadas por dos razones: la primera por su influencia demostrada en otras investigaciones nacionales e internacionales; y la segunda por la inexistencia de otras variables (figura judicial, el papel de los equipos psicosociales o el nivel educativo) que, si bien pueden ser de interés, no están incluidas en las bases de datos sobre divorcios y custodias.

4. Que el análisis se realice sobre el año 2017 encuentra su justificación en ofrecer al lector los datos más recientes disponibles. 
La utilización de esta fuente se justifica en que es la única que proporciona información diversa sobre nuestras variables objeto de estudio, ya que incluye ítems como la edad y nacionalidad de los cónyuges, número de hijos menores y mayores dependientes, demandante del proceso y tipo de custodia asignada, entre otras.

En relación a las técnicas multivariantes de análisis de datos utilizadas se emplea, en primera instancia, un análisis factorial de componentes principales (ACP), ya que facilita la interpretación de la realidad al menor coste posible en términos de pérdida de información (Ferrán, 1996) y conocer cómo se establecen determinadas relaciones entre los distintos factores. En segunda instancia, se realiza un análisis de correspondencias como aplicación particular del ACP, ya que resulta de gran utilidad para ver gráficamente las formas que adoptan las relaciones entre las categorías de las variables consideradas.

Para validar la adecuación de este análisis se aplica en primer lugar el test de esfericidad de Barlett, con el fin de detectar la posible correlación entre variables y en segundo lugar la medida Kaiser-Meyer-Olkin (KMO), con objeto de corregir posibles falsos resultados. Realizadas las pruebas pertinentes se procede al cálculo de la matriz de correlaciones utilizando el coeficiente de correlación producto momento de Pearson. Si bien es cierto que el análisis factorial, presenta dificultades a la hora de analizar variables dicotómicas, como es nuestro caso, la realidad es que nos permite dar respuesta a la necesidad de reducción de datos en nuestra investigación y en general en ciencias sociales. En este sentido, investigadores como Bartholomew (1980) no consideran la utilización del coeficiente de correlación de Pearson como un elemento problemático ya que las estimaciones obtenidas a partir de variables con distribución asimétrica no son severamente alteradas si los valores del índice de ajuste Root Mean Square Error (RMSEA) se mantienen dentro de los estándares aceptados $(0$ - 0,08) (Jöreskog, 2001).

El método utilizado para extraer los factores es el de componentes principales y se realiza una rotación Varimax, con el fin de mantener la interdependencia entre los factores rotados. Las variables seleccionadas pueden observarse en la tabla 2. Por último, precisar que todas las operaciones estadísticas se han realizado con el uso del software estadístico SPSS. 25.

Tabla 2. Variables utilizadas.

\begin{tabular}{|c|c|c|c|}
\hline Variables & Valores & Variables & Valores \\
\hline Tenencia de hijos menores & $\begin{array}{l}0-\text {-no } \\
1,2,3 \text { o más- Si }\end{array}$ & Custodia asignada & $\begin{array}{l}\text { Compartida } \\
\text { No compartida }\end{array}$ \\
\hline $\begin{array}{l}\text { Tenencia hijos mayores } \\
\text { dependientes }\end{array}$ & $\begin{array}{l}0-\text { no } \\
1,2,3 \text { o más- Si }\end{array}$ & & \\
\hline $\begin{array}{l}\text { Provincia (Producto Interior } \\
\text { Bruto) }\end{array}$ & $\begin{array}{l}\text { Almería, Cádiz, Córdoba, Granada, Huel- } \\
\text { va, Jaén, Málaga, Sevilla, }\end{array}$ & & \\
\hline Fallo de la sentencia & $\begin{array}{l}\text { 1-Consensuado } \\
\text { 2-No consensuado }\end{array}$ & & \\
\hline Nacionalidad varón & $\begin{array}{l}\text { 1-Española } \\
\text { 2-Extranjera }\end{array}$ & & \\
\hline Nacionalidad mujer & $\begin{array}{l}\text { 1-Española } \\
\text { 2-Extranjera }\end{array}$ & & \\
\hline Edad varón & 18 hasta +65 & & \\
\hline Edad mujer & 18 hasta +65 & & \\
\hline Demandante del proceso & $\begin{array}{l}\text { 1- Varón } \\
\text { 2- Mujer }\end{array}$ & & \\
\hline
\end{tabular}

Fuente: Elaboración propia a partir de la ENSD. 


\section{RESULTADOS Y DISCUSION}

A continuación, se presentan los resultados obtenidos tras aplicar las técnicas indicadas. En primer lugar, se muestra un análisis descriptivo de las variables utilizadas en el estudio (tabla 3), con el fin de mostrar al lector la información más completa posible.

De la tabla 3 se desprende como la mayoría de procesos de divorcio que se producen en Andalucía adquieren un carácter consensuado (72\%) y que, en más de la mitad de los casos, hay presencia de hijos o hijas menores (54\%). La demanda de divorcio suele ser interpuesta por ambos cónyuges, si bien en el caso de que sea solo uno de los miembros de la pareja el que lo demanda, la mujer lo hace con mayor frecuencia que el varón (22,9\% frente a 14,2\%). Estas disoluciones están caracterizadas, en su mayoría, en que ambos miembros de la pareja tengan nacionalidad española $(89,4 \%)$, siendo el porcentaje de divorcios binacionales relativamente bajo (7\%). Además, la edad media muestra una ligera diferencia situándose el varón en 48,3 años y la mujer en 44,8.

En segundo lugar, y con objeto de identificar empíricamente aquellos factores que tienen mayor peso a la hora de analizar el tipo de custodia asignada y que han sido investigados en la revisión de la literatura, se presentan los resultados del análisis factorial de componentes principales. Para comprobar que la realización del análisis factorial es adecuado se ha realizado el cálculo de la media de KMO de Kaiser (1970). Los valores de la prueba varían entre 0 y 1 , siendo el resultado obtenido de 0,517, por lo que resulta adecuada la realización del análisis factorial (Hair et al., 2005; Tabachnick \& Fidell, 2001). Además, la prueba de esfericidad de Bartlett indica que las variables están lo suficientemente intercorrelacionadas (Everitt y Wykes, 2001) para realizar el análisis factorial ( $p$-valor menor de 0,05 ).

Tal y como puede observarse en la tabla 4, los tres primeros componentes/factores extraídos para el caso de las disoluciones de los divorcios heterosexuales en Andalucía acumulan el $48,225 \%$ de la varianza total explicada.

Los resultados obtenidos mediante la aplicación de la rotación varimax (tabla 5) muestran como el fallo de la sentencia (inserto en el primer componente, 18,877\%), la tenencia y número de hijos menores y mayores dependientes (segundo componente, 15,371\%) y el producto interior bruto de cada provincia (tercer componente, 13,977\%) se encuentran estrechamente relacionados con la modalidad de custodia finalmente otorgada en Andalucía. Que estas variables aparezcan relacionadas con la asignación de la custodia encuentra relación en estudios revisados (indicados anteriormente), por lo que los resultados obtenidos adquieren robustez.

Además, este análisis factorial de componentes permite afirmar que los factores obtenidos son independientes, lo que posibilita profundizar un poco más en el estudio de las principales variables que componen los tres factores. Para ello se ha realizado un análisis de correspondencias que, aunque es una aplicación particular del ACP, ha sido de gran utilidad para ver gráficamente las formas que adoptan las relaciones entre las categorías de las variables utilizadas (ver figura 4).

El primer componente se encuentra integrado por una variable de carácter jurídico-legal, el fallo de la sentencia, que puede adoptar un carácter consensuado o contencioso. Para el caso Andaluz la mayoría de los procedimientos de divorcio son consensuados, $72 \%$ (ver tabla 1). El análisis de correspondencias realizado (gráfica 4) muestra que la modalidad de custodia exclusiva para la madre es la más repetida independientemente del fallo de la sentencia. Sin embargo, es preciso indicar que cuando el fallo de la sentencia es consensuado la posibilidad de que se otorgue la modalidad de custodia compartida aumenta. Sin embargo, cuando el proceso no es consensuado, la custodia exclusiva para la madre es la opción predominante, mientras que la custodia paterna obtiene un valor residual. En cuanto a otros tipos de custodia, cómo la asignada a otros familiares, sus valores son residuales. Estos hallazgos se encuentran en la línea de lo concluido en diversos estudios de ámbito nacional (Flaquer, 2015) e internacional (Jousselme, 2008) que apuntan que la existencia de consenso favorece la presencia de la modalidad compartida. Es de esperar que ante procesos consensuados las posibilidades de aplicar la custodia compartida aumenten.

En el segundo componente, que puede denominarse como número de hijos custodiados, se encuentran la tenencia y número de hijos menores o mayores dependientes. Esta variable ha sido señalada en multitud de investigaciones como clave para comprender los procesos de asignación de custodia y la modalidad finalmente asignada (Juby et al., 2005; Chausseborg et al., 2009). En Andalucía son más de la mitad los divorcios que incluyen hijos, ya sean menores o mayores dependientes, desde el comienzo de la serie histórica de la ENSD. 
Tabla 3. Análisis descriptivo de las variables utilizadas (2017).

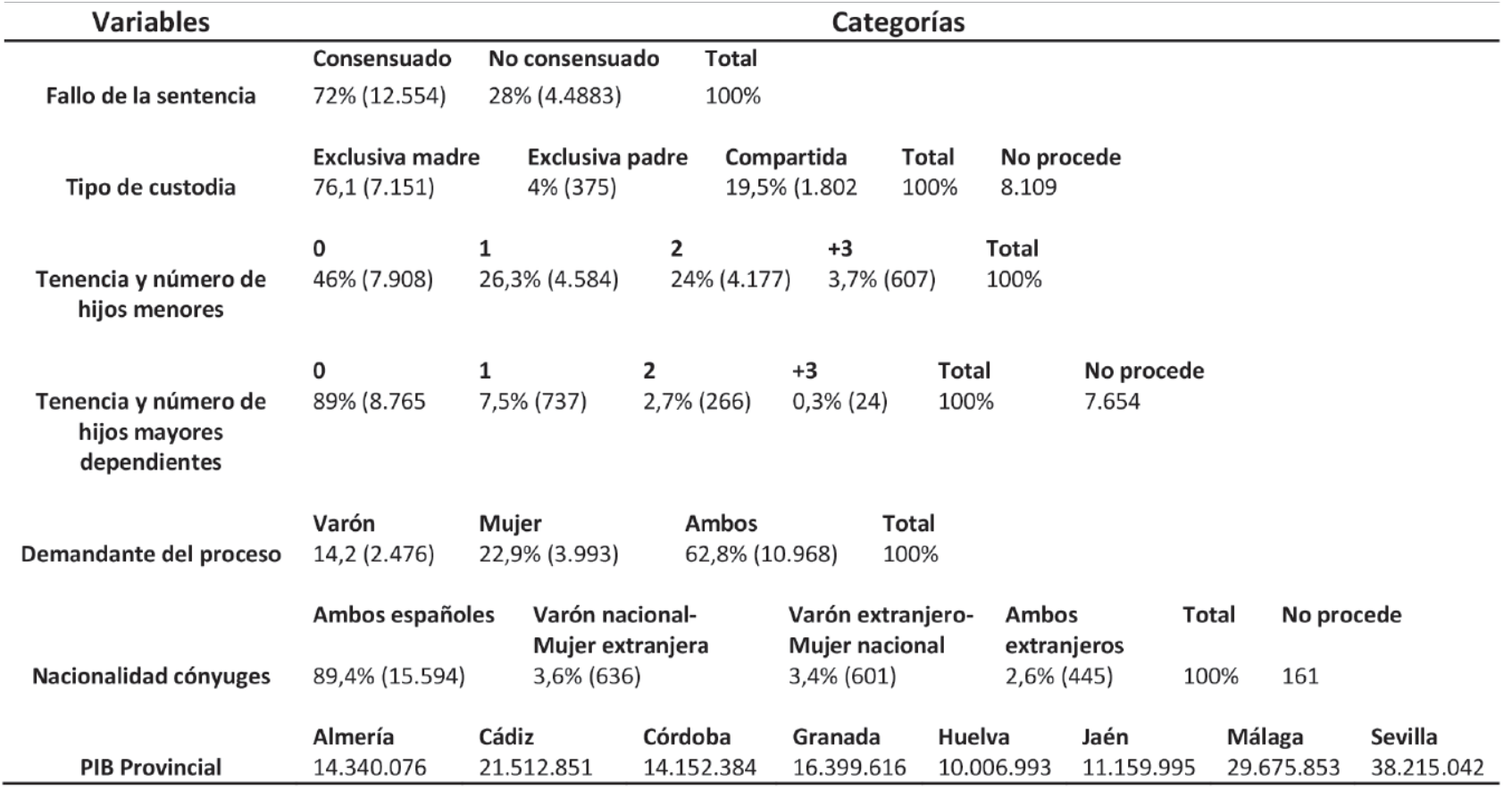

Fuente: Elaboración propia a partir de datos de la ENSD (2017).

Tabla 4. Prueba de KMO y Bartlett.

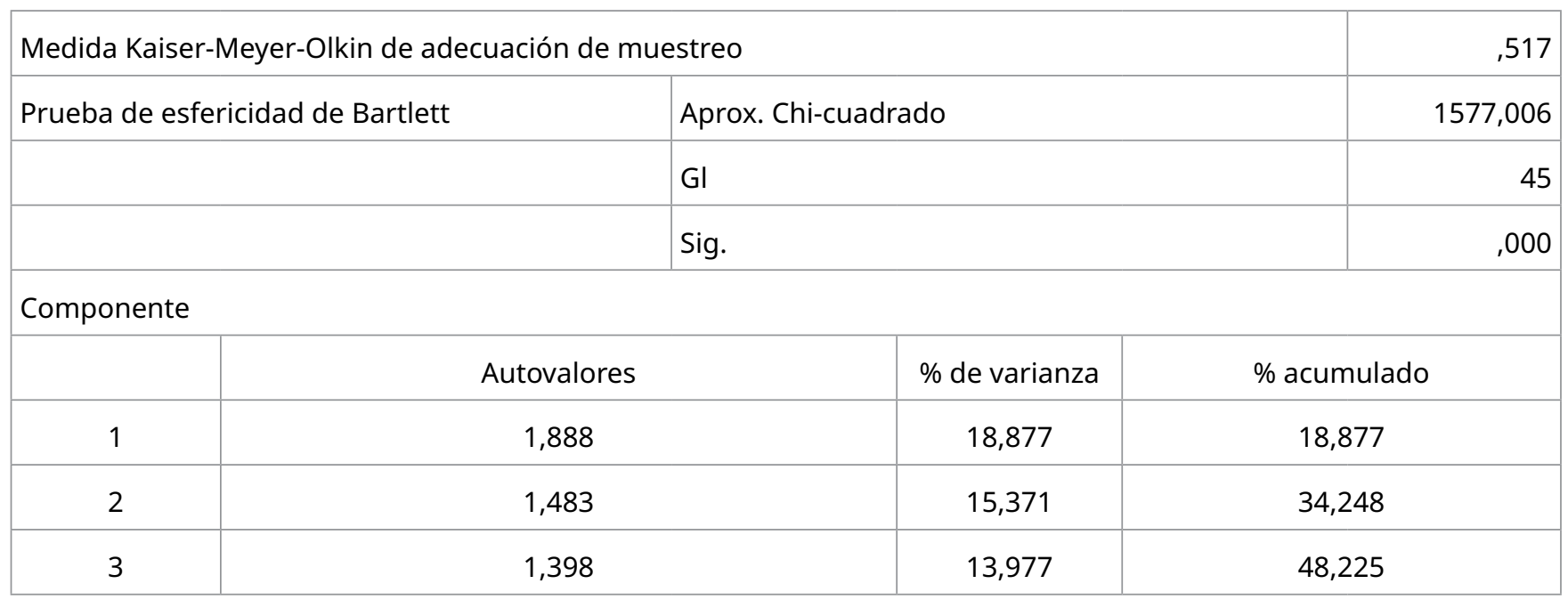

Fuente: Elaboración propia a partir de la ENSD (2017). 
Tabla 5. Matriz Componentes rotados.

\begin{tabular}{|c|c|c|c|}
\hline & \multicolumn{3}{|c|}{ Componente } \\
\hline & 1 & 2 & 3 \\
\hline PIB Provincia &,- 043 & ,097 & ,654 \\
\hline Edad varón &, 000 &,- 077 &,- 242 \\
\hline Edad mujer &,- 032 & ,069 &,- 300 \\
\hline Hijos menores &, 000 & ,802 &,- 073 \\
\hline Hijos mayores dependientes &,- 033 & ,716 & ,226 \\
\hline Fallo sentencia & ,731 &,- 015 & ,039 \\
\hline Demandante proceso & ,327 & 137 &,- 074 \\
\hline Nacionalidad varón &,- 024 &, 064 & 097 \\
\hline Nacionalidad mujer &,- 004 & 041 & ,123 \\
\hline
\end{tabular}

Fuente: Elaboración propia a partir de la ENSD (2017).

La relación entre el tipo de custodia y el número de hijos es significativa ( $p$-valor,000 es una constante), si bien es cierto que la modalidad exclusiva hacia la madre se muestra como la opción más repetida. Aparece como hecho destacable que cuando el número de hijos se sitúa en tres o más se incrementa la posibilidad de custodia compartida, siendo la custodia exclusiva para la madre más factible cuando existen uno o dos hijos. Este último hallazgo está relacionado con lo concluido por Jiménez y Becerril (2020) que señalan, para el caso de España, como la tenencia de uno o dos hijos está más relacionada con la custodia exclusiva para la madre. Este podría encontrar relación con el mayor esfuerzo que puede suponer un mayor número de hijos en cuanto al cuidado o la manutención, entre otros aspectos.

Resultaría interesante, poder comprobar si la edad de los hijos puede relacionarse con la modalidad de custodia finalmente sentenciada. En este sentido son diversas las investigaciones que han mostrado conclusiones interesantes (Maccoby \& Mnookin, 1992; Cancian \& Meyer, 1998). Sin embargo, con los datos disponibles en España en general, y para Andalucía concretamente, no se puede desagregar por grupos etarios y custodia, ya que la variable edad de los hijos no está presente en ninguna de las estadísticas disponibles. 


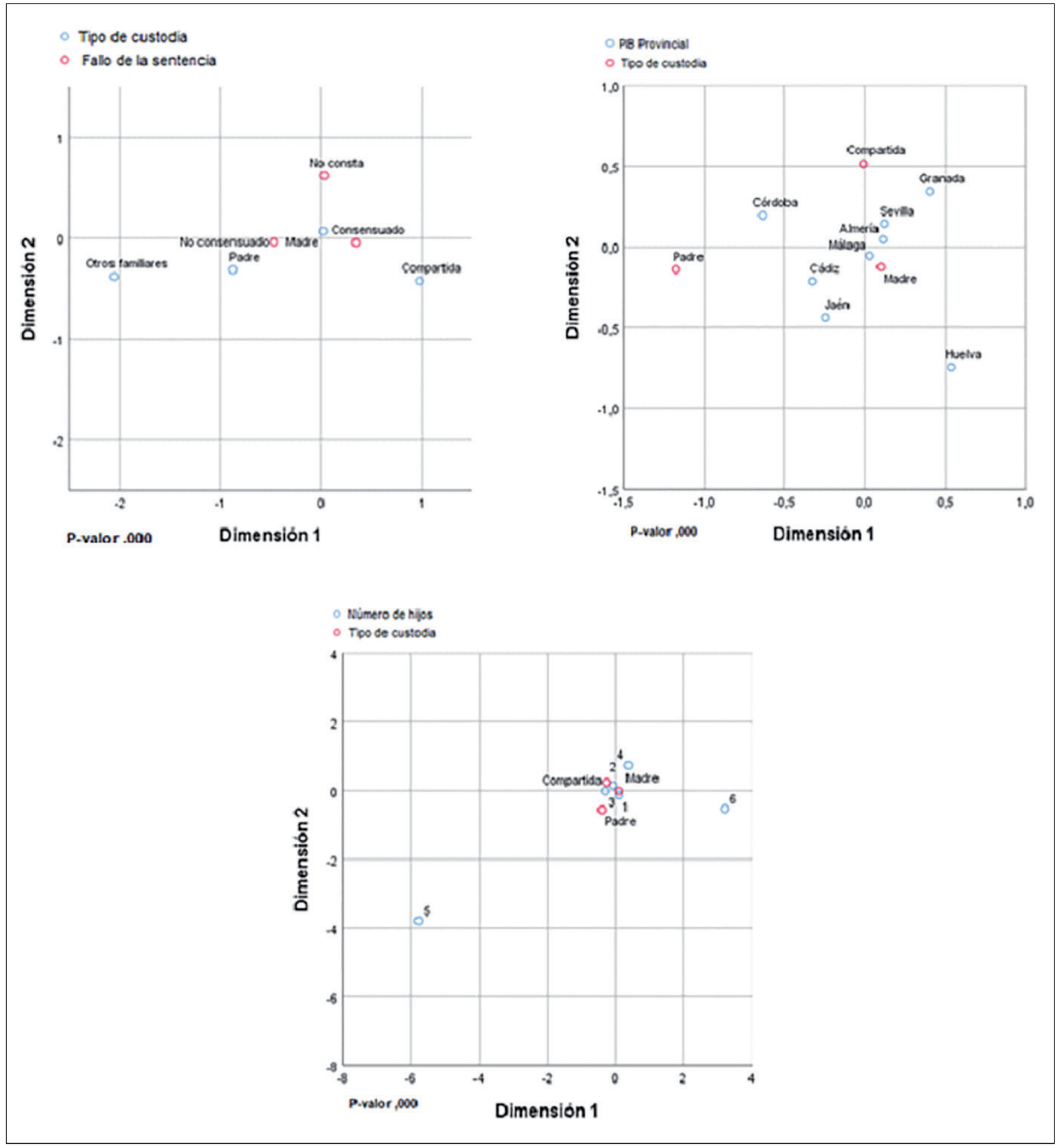

Figura 4. Análisis de correspondencias modalidad de custodia asignada por fallo de la sentencia, PIB provincial y número de hijos. Fuente: Elaboración propia a partir de la ENSD (2017).

Por último, el tercer componente, denominado económico, está integrado por el PIB. En este sentido, esta variable se encuentra relacionada con la modalidad de custodia finalmente asignada. Esta asociación permite apreciar un hecho relevante y bastante significativo en función de la posesión, por parte de cada provincia, de un mayor o menor PIB: aquellas provincias más alejadas de la modalidad de custodia compartida son, justamente, aquellas dos que poseen menor producto interior bruto (Huelva y Jaén). Esto está, en parte, relacionado con las tasas de empleo y desempleo de varones y mujeres en estas provincias. Atendiendo los datos de la Encuesta de Población Activa (2017) Jaén y Huelva han sido de las provincias andaluzas, 
junto a Cádiz, con menor tasa de actividad, en general. También se encuentran entre las que poseen mayor tasa de desempleo de la mujer. La importancia de estos aspectos está íntimamente relacionada con un aspecto clave para la asignación de la custodia: la capacidad de sustentabilidad de los cónyuges.

Aun así, las provincias andaluzas, en general y como sucede a nivel nacional, muestran un patrón similar: la custodia exclusiva hacia la figura materna es la modalidad más repetida (en todas se supera el 74\%, 2018) mientras que la compartida se mantiene en porcentajes que oscilan entre $17 \%$ de Cádiz y el $23 \%$ de Granada. Las variables de carácter económico-laboral, cómo indica la literatura, son importantes para comprender la modalidad finalmente establecida y, para el caso de Andalucía, el PIB lo es como indica su relación estadística.

Sin embargo, variables como la edad o nacionalidad de los cónyuges y el demandante del proceso parecen guardar menor relación con la modalidad asignada, al menos para el caso de Andalucía, como puede visualizarse en el análisis ejecutado. De esta forma, la hipótesis establecida: la tenencia y número de hijos menores y mayores dependientes, la edad de los miembros de la pareja y el fallo de la sentencia serán las variables claves para comprender cómo se produce la asignación de la custodia de los hijos en Andalucía, queda parcialmente validada. Esto se debe a que el PIB de cada una de las provincias se muestra más decisivo, y tiene mayor peso, en relación a la modalidad asignada que la edad de los miembros de la pareja.

\section{CONCLUSIONES}

El interés principal de esta investigación reside en que, si bien la asignación de la custodia de los hijos tras la disolución de matrimonios heterosexuales, ha sido objeto de estudio a nivel nacional no existe ningún análisis pormenorizado para Andalucía. Sobre todo, si tenemos presente que más de la mitad de los divorcios que se producen en esta comunidad involucran la presencia de hijos e hijas. Para ello se ha utilizado metodología cuantitativa mediante la explotación de datos secundarios que provienen de la Estadística de Nulidades, Separaciones y Divorcios (2007-2017).

Las principales conclusiones extraídas radican en que el fallo de la sentencia, la tenencia y número de hijos menores o mayores dependientes y el PIB provincial guardan estrecha relación con la modalidad de custodia asignada. Otras variables como la edad y nacionalidad de los miembros del matrimonio divorciado o el demandante del proceso presentan menor relación con el hecho de que se otorgue la custodia de forma exclusiva, hacia el padre o madre, o compartida.

Concretamente, con respecto al fallo de la sentencia, los procesos consensuados conducen, de forma más probable, a la custodia compartida. Sin embargo, aunque estos suponen más del $70 \%$ de los casos totales, la tendencia a otorgar la custodia de los hijos a la madre sigue predominando.

En cuanto a la tenencia y número de hijos menores o mayores dependientes, se observa que el número de estos influye significativamente en el tipo de custodia. En este sentido cuando se habla de un número de hijos no muy numeroso (1-2) la correspondencia más directa se produce con la modalidad exclusiva hacia la madre. Sin embargo, cuando existe número de hijos amplío (más de tres) la relación con la custodia compartida es más cercana. Esto puede deberse, entre otros aspectos, en el condicionamiento e implicaciones que supone el cuidado de un mayor número de hijos e hijas en el resto de ámbitos (laboral, educativo, ocio y tiempo libre, entre otros).

El Producto Interior Bruto, aparece asociado a la modalidad de custodia que se asigna, siendo destacable cómo justamente las dos provincias con PIB más bajo, Huelva y Jaén, se encuentran más alejadas de la modalidad compartida. Esto, encuentra relación con las tasas de empleo y desempleo de varones y mujeres en las diferentes provincias andaluzas.

La presente investigación muestra algunas limitaciones, ya que dejan fuera de los análisis variables sociodemográficas como la edad de los hijos o el nivel de ingresos de los cónyuges; y variables de tipo contextual como la relevancia de la figura judicial y de los equipos psicosociales. Estas variables pueden arrojar luz para una comprensión más global y completa de la modalidad de custodia finalmente asignada. Las razones por las que no se han incluido son principalmente dos: la no inclusión de estos ítems en las bases de datos y en la inexistencia de estudios pormenorizados para el caso de Andalucía sobre esta temática. 
Por todo lo analizado y expuesto queda patente la necesidad de prestar una mayor atención a un fenómeno social que implica a una parte importante de la población andaluza. La consideración de las variables ya señaladas y la realización de estudios cualitativos, pueden permitir comprender de una manera más profunda este fenómeno y repercutir positivamente en la puesta en marcha de una regulación autonómica y de políticas públicas adecuadas. Además, el análisis de otro tipo de vínculos, y su ruptura, tales como parejas de hecho o la disolución de matrimonios de personas del mismo sexo podrían plantarse como una línea de investigación complementaria.

\section{Agradecimientos}

Al Ministerio de Ciencia, Innovación y Universidades ya que la presente investigación cuenta con financiación del programa para la ayuda de formación de profesorado universitario (FPU).

\section{Declaración responsable y conflicto de intereses}

La autora y los autores del presente artículo declaran que en la actualidad no existe ningún conflicto de intereses en relación a la publicación de este artículo y se comprometen a comunicar cualquier potencial conflicto. Asimismo, se informa que las tareas de investigación llevadas a cabo (marco teórico, metodología, validación, análisis de los datos, redacción de resultados, conclusiones y revisión final del texto) han sido realizadas conjuntamente por la autora y autores.

\section{REFERENCIAS}

Alberdi, I. (1979) Historia y sociología del divorcio. Centro de Investigaciones Sociológicas.

Ayuso, L. (2019). Nuevas imágenes del cambio familiar en España. Revista Española de Sociología, 28(2), 269-287. https:// doi.org/10.22325/fes/res.2018.72

Bartholomew, D. (1980). Factor analysis for categorical data. Journal of the Royal Statistical Society. Series B (Methodological), 42(3), 293-321. https://doi.org/10.1111/j.2517-6161.1980.tb01128.x

Becerril, D. \& Jiménez, J. (2019). Reformas legales y su efecto sobre las rupturas matrimoniales. OBETS. Revista de Ciencias Sociales, 14(2), 287-311. https://doi.org/10.14198/OBETS2019.14.2.01

Becerril, D. \& Jiménez, J. (2016). Acuerdos y desacuerdos: conflictividad en las rupturas de parejas homos y heterosexuales. En Becerril, D, y Lozano, A. (ed). Sociología del conflicto en las sociedades contemporáneas. (41-58). Dykinson.

Becerril, D. (2015). La ruptura matrimonial en España. En A. Torres (ed.), España 2015. Situación social. Centro de Investigaciones Sociológicas.

Becerril, D. (2014). Cortes Generales y custodia compartida en España. En A.Pérez, N. Castillo \& F. Estrena (eds.), Gestión de procesos sociales. La experiencia de Cuba, España y México. Fundación Cronos, Vida y Cultura.

Becerril, D. (2008). La percepción social del divorcio en España. Revista Española de Investigaciones Sociológicas (Reis), 123, 187-208. https://doi.org/10.2307/40184897

Becerril, D. (1999). Después del divorcio. Centro de Investigaciones Sociológicas.

Borrajo, S. (1989) La ruptura matrimonial en España. Madrid: Eudema

Brinig, M. \& Allen, D. (2002). These boots are made for walking: why most divorce filers are women. American Law and Economics Review, 2(1), 126-169. https://doi.org/10.1093/aler/2.1.126

Cancian, M. \& Meyer, D. (1998). Who gets custody? Demography, 35, 147-157. https://doi.org/10.2307/3004048

Catalán, M. (2011). La custodia compartida. Revista de Derecho y Criminología. http://repositorio.ucam.edu/bitstream/ handle/10952/574/La\%20custodia\%20compartida.\%20Mar\%c3\%ada\%20Jos\%c3\%a9\%20Catal\%c3\%a1n\%20Fr\%c3\%adas.pdf?sequence=1\&isAllowed=y 
Catalán, M., Peña, B., Alemán, S., Aragón, C., García, V., Marín, M., Matas, C. \& Concepción, M. (2007) La custodia compartida: concepto, extensión y bondad de su puesta en escena. Debate entre Psicología y Derecho. Anuario de Psicología Jurídica, 17(1), 131-151.

Chaussebourg, L., Carrasco, V. \& Lermenier, A. (2009). Le divorce. Ministere de la Justice.

Everitt, B. \& Wykes, T. (2001). Diccionario de Estadística para Psicólogos. Ariel.

Ferrán, M. (1996). SPSS para Windows 95. Programación y análisis estadístico. McGraw-Hill.

Flaquer, L. (2012). Liberalización sin protección: Un balance de los resultados de la aplicación de la Ley de divorcio de 2005. En Picontó, T. (ed.) La Custodia Compartida a debate (23-44). Dykinson.

Flaquer, L. (2015), El avance hacia la custodia compartida o el retorno del padre tras una larga ausencia. En Torres, C. (ed.) España 2015. Situación social (351-359) Centro de Investigaciones sociológicas.

Hair, J., Anderson, R., Tatham, R. \& Black, W. (2005). Multivariate data analysis. Prentice All International.

Jiménez, J. \& Becerril (2020). Main Characteristics Associated with the Assignment of Custodies After the Divorce. Journal of Divorce \& Remarriage, 61(8), 615-635. https://doi.org/10.1080/10502556.2020.1827344

Jöreskog, K. (2001). Analysis of ordinal variables 2. Cross-sectional data. Structural Equation Modelling with Lisrel 8.51. Friedrich Schiller: Universitat, Jena.

Jousselme, C. (2008). Résidence alternée:Pour/Contre. Archives de Pediatrie, 15, 483-485. https://doi.org/10.1016/S0929693X(08)71805-0

Juby, H., Le Bourdais, C. \& Marcil-Gratton, N. (2005). Sharing roles, sharing custody? Couples' characteristics and children's living arrangements at separation. Journal of Marriage and Family, 67(1), 157-172. https://doi.org/10.1111/ j.0022-2445.2005.00012.x

Kaiser, H. (1970). A second generation little jiffy. Psychometrika, 35(4), 401-415. https://doi.org/10.1007/BF02291817

Lezcano, R. (1979) El divorcio en la segunda república. Akal

López-Pintor, R. \& Toharia, J. (1989). Separación y divorcio en España. Ministerio de Trabajo y Seguridad Social

Maccoby, E. \& Mnookin, R. (1992). Dividing the child: Social and legal dilemmas of custody. Harvard University Press.

Meil, G. (2015). Familia. En Torres, C. (ed.) España 2015, situación social. Centro de Investigaciones Sociológicas.

Morillas, M. (2016) Régimen jurídico de la custodia compartida: el interés del menor. En Becerril, D. \& Venegas, M. (eds.). La custodia compartida en España. Dykinson.

Prieto, P. (2014). Custodia compartida en Aragón: diferencias con otras Comunidades Autónomas y análisis jurisprudencial [Trabajo Fin de Grado, Universidad de Zaragoza] https://zaguan.unizar.es/record/15796/files/TAZ-TFG-2014-1401

Rapoport, B. \& Le Bourdais, C. (2001). Temps et formes padres familiales. Loisir et société, 2(24), 585-617. https://doi. org/10.7202/000196ar

Ravetllat, I. (2012). El interés superior del niño: concepto y delimitación del término. Educatio Siglo XXI, 30(2), 89-108.

Ruiz, R. \& Alcázar, R. (2017) Factores determinantes en la atribución de la custodia compartida. Un estudiosociológico den loa Juzgados de Familia. En Becerril, D. \& Venegas, M. (Eds.), La custodia compartida en España (109-124). Dykinson.

Solsona, M. \& Simó, C. (2007). Evolución histórica del divorcio en España desde la aprobación de la ley de 1981 hasta la reforma en 2004. En Cabré, A. y Miret, P. (Eds.) La constitución familiar en España: Tendencias y factores. Fundación BBVA

Solsona, M. (2015) Divorcio, generaciones y género. En Torres, C. (Ed) España 2015. Situación social (117-126). Madrid: Centro de Investigaciones sociológicas.

Solsona, M., Spijker, J. \& Ajenjo, M. (2017). Calidoscopio de la custodia compartida en las regiones de España. En Becerril, D. \& Venegas, M. (eds.), La custodia compartida en España. (45-72). Dykinson.

Solsona, M. Brullet, C. \& Spijker, J. (2014). Coparentalitat i custòdia compartida a Catalunya. Documents d'Anàlisi Geogràfica, 60(2), 387-415. https://doi.org/10.5565/rev/dag.137

Solsona, M. \& Simó, C. (2007). Evolución histórica del divorcio en España desde la aprobación de la ley de 1981 hasta la reforma en 2004. En Cabré, Ana. \& Miret, P. (eds.), La constitución familiar en España: Tendencias y factores. Fundación BBVA

Spijker, J. (2012). Trends in custody arrangements in Spain since the Divorce Reform of 2005. Papers de Demografia, 404.

Suso, A., González, I., Pérez, A. \& Velasco, M. (2012). Análisis de los modelos de custodia derivados de las situaciones de separación y divorcio en España. Ministerio de sanidad, servicios sociales e igualdad.

Tabachnick, B. \& Fidell, L. (2001). Using multivariate statistics. Allyn and Bacon. 
Torres-Perea, J. (2011) Custodia compartida: Una alternativa exigida por la nueva realidad Social. Indret: Revista para el análisis del Derecho, 4.

Viñas, D. (2012). Medidas relativas a los hijos menores en caso de ruptura. Especial referencia a la guarda. Revista para el análisis del Derecho, 3, 2-55.

Wilcox, K., Wolchik, S. \& Braver, S. (1998). Predictors of maternal preference for joint or sole legal custody. Family Relations, 47(1), 93-101. https://doi.org/10.2307/584855 